\title{
E1 desarrollo sostenible y los derechos culturales
}

Sustainable Development and Cultural Rights

\section{Carlos J. Villaseñor Anaya Afiliación}

\author{
«La creatividad es colectividad. \\ Más que una expresión de individualidad, \\ la creatividad toma forma en un contexto social» \\ S. Alexander HASLAM ${ }^{1}$
}

\section{Del desarrollo cultural sostenible, algunos conceptos}

Desde la perspectiva del desarrollo cultural sostenible, los procesos económicos y los rendimientos que ellos puedan producir no son un fin en sí mismos ${ }^{2}$, sino solamente una de las variables interdependientes sobre las cuales se hace posible satisfacer las necesidades culturales de la generación presente, sin

\footnotetext{
1 Haslam, Alexander, et al. Scientific American MIND, Vol. 25, número 4, agosto 2014, p. 31.

2 Ya Aristóteles (384 a. C. a 322 a. C), cuando hablaba de los Modos de Vida, indica que la riqueza no es, desde luego, el bien que buscamos, pues no es más que un instrumento para conseguir algún otro fin (Ética Nicomáquea, Libro 1, Capítulo 5). En el texto vinculamos la obtención de la riqueza con el objetivo de alcanzar el desarrollo sostenible, en los términos en que esta conceptualizado en el informe de la Comisión Mundial sobre el Medio Ambiente y el Desarrollo, 1987, titulado «Nuestro Futuro Común». Consta de tres pilares, el desarrollo sostenible trata de lograr, de manera equilibrada, el desarrollo económico, el desarrollo social y la protección del medio ambiente.
} 
comprometer la capacidad de las generaciones futuras para continuar desarrollándose a partir del conjunto de los rasgos distintivos, espirituales y materiales, intelectuales y afectivos que constituyen su manera más lograda de estar en el mundo.

En el párrafo anterior aparecen dos conceptos básicos. El primero de ellos es el del desarrollo sostenible, en los términos en que esta conceptualizado en el informe de la Comisión Mundial sobre el Medio Ambiente y el Desarrollo (1987), titulado «Nuestro Futuro Común»; y que sería retomado en el documento titulado «Transformar nuestro mundo: la Agenda 2030 para el Desarrollo Sostenible», que fue aprobado por la Asamblea Genera de la Organización de las Naciones Unidas, el 25 de septiembre del $2015^{3}$.

El gran cambio de paradigma que, a mi juicio, establece la Agenda 2030 es que propone 17 objetivos y 169 metas, para cuya consecución se deberán conjugar armónicamente las tres dimensiones del desarrollo sostenible: la económica, la social y la ambiental. Es decir que ninguna de las tres vertientes debe crecer independientemente, por encima o a costa de las otras, pues en ese momento dejaría de ser sostenible.

Para ser muy claro, con base en dicha conceptualización, el crecimiento económico no puede florecer con independencia de las vertientes social y ambiental. Para ser sostenible, si bien es indispensable la existencia de una vertiente económica sólida y viable, esta debe coexistir en armonía con los aspectos social y ambiental.

Durante las discusiones hacia lo que sería la Agenda 2030, específicamente en el Congreso Internacional sobre: La cultura, clave para el desarrollo sostenible, que se llevó a cabo en

3 El documento completo «Transformar nuestro mundo: la Agenda 2030 para el Desarrollo Sostenible», se puede consultar en: https://www.un.org/ga/search/view_doc.asp?symbol=A/ RES $/ 70 / 1 \&$ Lang $=S$.

Villaseñor Anaya, Carlos J. (2021). «El desarrollo sostenible y los derechos culturales». Monograma. Revista Iberoamericana de Cultura y Pensamiento, n. ${ }^{\circ}$ 9, pp. 393-405. doi: 10.36008/monograma.2021.09.2249. http: revistamonograma.com. ISSN: 2603-5839. 
Hangzhou, China, en el $2013^{4}$, se propuso la inclusión de un objetivo específico centrado en la cultura, basado en el patrimonio, la diversidad, la creatividad y la transmisión del conocimiento, y provisto de metas e indicadores claros que vincularan la cultura a todas las dimensiones del desarrollo sostenible.

Si bien es cierto que en la Agenda 2030 de la ONU no se incluyó un objetivo específico centrado en la cultura, es indudable que lo cultural está implícito a lo largo de la misma, ya que resulta transversal a todos los objetivos y metas que la conforman. Ninguno de los objetivos y metas puede ser abordado dejando fuera la perspectiva cultural.

¿En qué sentido es que la cultura está implícita en el desarrollo sostenible? Es aquí a donde entra el segundo concepto al que me he referido en el primer párrafo. Hagamos un poco historia.

La Unesco reconoce a la Declaración de México sobre las Políticas Culturales como aquel documento, suscrito por 144 países, que sienta las bases que dan inicio a una discusión global sobre la relación entre cultura y desarrollo.

Por la influencia de los movimientos sociales iniciados en el año 1968 y que estuvieron muy activos durante la década de los años 1970, en Declaración de México de 1982 se alcanzó un importante avance al reconocer que «1. Cada cultura representa un conjunto de valores único e irreemplazable, ya que las tradiciones y formas de expresión de cada pueblo constituyen su manera más lograda de estar presente en el mundo». Es decir, que la cultura va mucho más allá de solamente las bellas artes y el patrimonio cultural, para constituirse en el cristal a través del cual vemos, interpretamos, interactuamos y aportamos al flujo de lo simbólico.

\footnotetext{
4 El documento completo de la «Declaración de Hangzhou, Situar la cultura en el centro de las políticas de desarrollo sostenible» se puede consultar en: https://unesdoc.unesco.org/ ark:/48223/pf0000221238.
}

Villaseñor AnAYA, Carlos J. (2021). «El desarrollo sostenible y los derechos culturales». Monograma. Revista Iberoamericana de Cultura y Pensamiento, n. ${ }^{\circ}$ 9, pp. 393-405. doi: 10.36008/monograma.2021.09.2249. http: revistamonograma.com. ISSN: 2603-5839. 
Ya lo describía atinadamente el poeta Argentino Hilario Ascasubi: «Las cosas no se ven como son. Las vemos como somos».

En consecuencia, es también en esa Declaración en la que se incluye lo que, hasta la fecha, es la definición operativa de cultura que utiliza la Unesco:

en su sentido más amplio, la cultura puede considerarse actualmente como el conjunto de los rasgos distintivos, espirituales y materiales, intelectuales y afectivos que caracterizan a una sociedad o un grupo social. Ella engloba, además de las artes y las letras, los modos de vida, los derechos fundamentales al ser humano, los sistemas de valores, las tradiciones y las creencias.

Es precisamente desde esa perspectiva que conceptualizamos que el desarrollo cultural sostenible es aquel que permite a las generaciones futuras seguir aprovechando los rasgos distintivos, espirituales y materiales, intelectuales y afectivos de su identidad social e individual.

Desde luego no estamos hablando de los valores de una cultura hegemónica, sino de las múltiples expresiones de la creatividad humana, en sus relaciones sociales y con el ambiente.

$\mathrm{Al}$ respecto resulta oportuno recordar el contenido de la Declaración Universal a favor de la Diversidad Cultural (Unesco, 2001) que, en su artículo 1 nos recuerda:

La cultura toma diversas formas a través del tiempo y del espacio. Esta diversidad se manifiesta en la originalidad y la pluralidad de identidades que caracterizan los grupos y las sociedades que componen la humanidad. Fuente de intercambios, innovación y creatividad, la diversidad cultural es, para el género humano, tan necesaria como la diversidad biológica para los organismos vivos. En este sentido, constituye patrimonio

Villaseñor Anaya, Carlos J. (2021). «El desarrollo sostenible y los derechos culturales». Monograma. Revista Iberoamericana de Cultura y Pensamiento, n. ${ }^{\circ}$ 9, pp. 393-405. doi: 10.36008/monograma.2021.09.2249. http: revistamonograma.com. ISSN: 2603-5839. 
común de la humanidad y debe reconocerse y consolidarse en beneficio de las generaciones presentes y futuras.

Es así que podemos concluir que, la promoción y salvaguardia de la diversidad cultural, es un elemento fundamental del desarrollo cultural sostenible.

\section{De la economía de la cultura a la economía creativa}

Es también en la Declaración de México de 1982 en la que se da entrada a la vertiente económica del desarrollo cultural, cuando se habla de las industrias culturales.

La perspectiva desde la que la Declaración de 1982 se aproxima a ellas es la de que las industrias culturales (párrafos 38 y 39) habían adquirido un importante papel como portadoras de contenidos simbólicos (lo cual no ha hecho más que incrementarse a lo largo de los años); de donde hay que estar atentos a que las industrias culturales de los países ricos no desplacen los contenidos simbólicos de los países en desarrollo, y hacer lo necesario para que las industrias culturales de estos países tengan una adecuada presencia en el flujo mundial. Sobretodo, con el propósito de garantizar la diversidad de las expresiones culturales, como fuente de la creatividad de la especie humana.

Sobre esa bases es que se construiría la Convención Unesco para la Protección y Promoción de la Diversidad de las Expresiones Culturales, con la cual se busca garantizar la diversidad de los contenidos simbólicos como medio para salvaguardar la creatividad de la especie humana; donde la economía de la cultura juega un papel importante en el desarrollo, pero no es el fin del mismo, sino solamente una de sus herramientas. El propósito es diversificar y ampliar el repertorio cultural de la humanidad, en aras de salvaguardar la creatividad de la especie humana. 
Habitualmente se reconoce al libro The Creative Economy: How People Make Money From Ideas ("La economía creativa: transformar una idea en beneficios») del autor John Howkins, publicado en 2001, como uno de los principales fundamentos del concepto de economía creativa. En términos generales, el libro reconoce que si bien la creatividad es una capacidad universal, es solamente aquella que se hace aprovechable a través de los instrumentos de la propiedad intelectual y/o industrial, la que se convierte en motor de un nuevo y prometedor mercado. Howkins es también muy puntual en señalar que los contenidos de la economía creativa se caracterizan por ser —al mismo tiempo- bienes económicos y portadores de valores simbólicos.

Otra vertiente de la economía creativa, más cercana al espíritu de la Declaración de México de 1982, es la que surge en en el año 2002, cuando la Conferencia de las Naciones Unidas para el Comercio y el Desarrollo (UNCTAD) comienza a analizar la conveniencia de establecer un programa específico para medir el impacto económico de la economía creativa, a efecto de evaluar su importancia como una nueva alternativa para el fomento al desarrollo. Es allí donde, por primera vez, se entrelazan los conceptos economía creativa y desarrollo. Como consecuencia de lo anterior, en el 2004 (São Paulo, Brasil) se conjunta un primer Grupo de Alto Nivel Sobre las Industrias Creativas y el Desarrollo.

El producto de los trabajos de ese Grupo de Alto Nivel, quedó plasmado en el llamado Consenso de São Paulo (UNCTAD $\mathrm{XI})$ que, entre otras conclusiones, expresa que: «Las industrias creativas pueden contribuir a promover externalidades positivas, al ayudar a conservar y promover el patrimonio y la diversidad culturales. Aumentar la participación de los países en desarrollo, en las oportunidades nuevas y dinámicas de crecimiento del comercio mundial, y en las ventajas que de ello se derivan, para obtener beneficios en materia de desarrollo, a partir del comercio internacional y las negociaciones comerciales. Es importante y

Villaseñor Anaya, Carlos J. (2021). «El desarrollo sostenible y los derechos culturales». Monograma. Revista Iberoamericana de Cultura y Pensamiento, n. ${ }^{\circ}$ 9, pp. 393-405. doi: 10.36008/monograma.2021.09.2249. http: revistamonograma.com. ISSN: 2603-5839. 
representa un juego de suma positiva para los países desarrollados y los países en desarrollo».

También a partir del 2004, queda instalado el Programa de Economía Creativa, como parte de las actividades regulares de la Conferencia de las Naciones Unidas Para el Comercio y el Desarrollo (UNCTAD), que presentaría dos Reportes de Economía Creativa (2008 y 2010) y, un tercero, en conjunto con la Unesco (2013).

En el Reporte de Economía Creativa 2010 (UNCTAD) encontramos un gráfico en el que se resume que la Economía Creativa está integrada por los sectores de la economía de la cultura (patrimonio y artes), las industrias culturales (medios) y las industrias creativas (creaciones funcionales), los cuales están integrados como tres círculos concéntricos de un mismo cuerpo total, con lo cual queda definitivamente establecido el concepto de economía creativa.

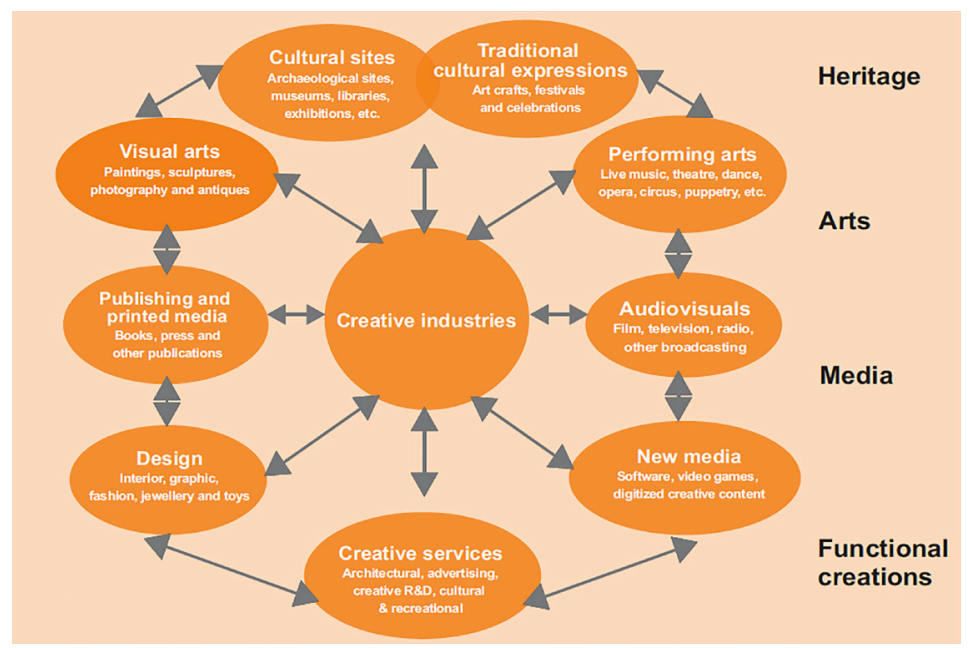

FIGURA 1. Clasificación de las industrias creativas.

Fuente: Reporte de Economia Creativa 2010 (UNCTAD), p. 37

https://unctad.org/system/files/official-document/ditctab20103_en.pdf.

Villaseñor Anaya, Carlos J. (2021). «El desarrollo sostenible y los derechos culturales». Monograma. Revista Iberoamericana de Cultura y Pensamiento, n. ${ }^{\circ}$ 9, pp. 393-405. doi: 10.36008/monograma.2021.09.2249. http: revistamonograma.com. ISSN: 2603-5839. 
Cierro el presente apartado recordando la histórica resolución aprobada por la Asamblea General de la ONU, en el año 2010, en la que se reconoce que:

...la cultura es un componente esencial del desarrollo humano, constituye una fuente de identidad, innovación y creatividad para las personas y para la comunidad y es un factor importante en la lucha contra la pobreza al promover el crecimiento económico y la implicación en los procesos de desarrollo. ${ }^{5}$

\section{De los derechos culturales como derechos humanos}

Ya desde la Declaración Universal De Los Derechos Humanos, adoptada y proclamada por la Asamblea General de la Organización de las Naciones Unidas, el 10 de diciembre de 1948, se «garantiza a toda persona el derecho a obtener, habida cuenta de la organización del Estado, la satisfacción de sus derechos culturales y a tomar parte libremente en la vida cultural de la comunidad».

Sin demérito del valor moral de esa Declaración, el Pacto Internacional De Derechos Económicos, Sociales Y Culturales (PIDESC) (1966), es reconocido por la ONU como un Tratado en materia de derechos humanos ${ }^{6}$, por lo que vincula jurídicamente a los países que lo han suscrito y ratificado. En él se establece lo siguiente:

Artículo 15

1. Los Estados Partes en el presente Pacto reconocen el derecho de toda persona a:

a) Participar en la vida cultural;

5 Resolución AG/65/166. Cultura y desarrollo. Resolución aprobada por la Asamblea General de la ONU, 20 de diciembre de 2010. Puede ser consultada en: https://www.un.org/en/ga/ search/view_doc.asp?symbol=A/RES/65/166\&Lang=S.

$6 \mathrm{La}$ referencia que la $\mathrm{ONU}$ lo reconoce como un tratado en materia de derechos humanos se puede consultar en la siguiente página: https://www.ohchr.org/sp/professionalinterest/pages/ coreinstruments.aspx.

Villaseñor Anaya, Carlos J. (2021). «El desarrollo sostenible y los derechos culturales». Monograma. Revista Iberoamericana de Cultura y Pensamiento, n. ${ }^{\circ}$ 9, pp. 393-405. doi: 10.36008/monograma.2021.09.2249. http: revistamonograma.com. ISSN: 2603-5839. 
b) Gozar de los beneficios del progreso científico y de sus aplicaciones;

c) Beneficiarse de la protección de los intereses morales y materiales que le correspondan por razón de las producciones científicas, literarias o artísticas de que sea autora.

2. Entre las medidas que los Estados Partes en el presente Pacto deberán adoptar para asegurar el pleno ejercicio de este derecho, figurarán las necesarias para la conservación, el desarrollo y la difusión de la ciencia y de la cultura.

3. Los Estados Partes en el presente Pacto se comprometen a respetar la indispensable libertad para la investigación científica y para la actividad creadora.

El hecho de que el PIDESC sea reconocido como un Tratado en materia de derechos humanos tiene numerosas consecuencias prácticas:

1. Los derechos en él establecidos son reconocidos como derechos humanos. En ese orden de ideas, todos y cada uno de los derechos establecidos en el artículo 15, en materia cultural, tienen ese rango.

2. El seguimiento y asesoría para el mejor cumplimiento de todos los Tratados en materia de derechos humanos están bajo la autoridad del Consejo de Derechos Humanos y la oficina del Alto Comisionado de las Naciones Unidas quienes, a solicitud de quien considere que sus derechos humanos han sido violados, puede iniciar un Procedimiento Especial, que tiene por objeto investigar la presunta violación y hacer recomendaciones para que el Estado Parte pueda restituir el estado de cumplimiento de los derechos humanos a los que se obligó mediante la ratificación del PIDESC.

3. Cada uno de los Tratados en materia de Derechos Humanos tiene un órgano específico de seguimiento e in- 
terpretación. En el caso del PIDESC es el Comité de los Derechos Económicos; Sociales y Culturales (CDESC). ${ }^{7}$

4. El CDESC emite Observaciones Generales que profundizan sobre el alcance de los derechos reconocidos en el PIDESC. A la fecha, en relación con el artículo 15. ${ }^{\circ}$ del PACTO, ha emitido las Observaciones Generales 17 y $21^{8}$.

5. Desde el año 2009, el Consejo de los Derechos Humanos decidió crear un nuevo procedimiento especial titulado «Experto independiente en la esfera de los derechos culturales».9, quien ha venido emitiendo Informes específicos sobre diversos temas vinculados a los derechos culturales.

Esta breve relación de lo que constituye el sistema multinacional de los derechos culturales, busca hacer evidente que los tres apartados en los que se divide el artículo 15 son parte integrante de los derechos humanos reconocidos por la inmensa mayoría de los países; y, al igual que los demás derechos humanos, «son universales, indivisibles e interdependientes; de donde su promoción y respeto cabales son esenciales para mantener la dignidad humana y para la interacción social positiva de individuos y comunidades en un mundo caracterizado por la diversidad y la pluralidad cultural» $»^{10}$.

Si bien es cierto que la promoción de las artes y la preservación del patrimonio cultural han sido objeto de la políticas públicas

\footnotetext{
7 Para conocer más sobre las actividades del Comité puede acudir a la pagina: https://www. ohchr.org/SP/hrbodies/cescr/pages/cescrindex.aspx.

8 Para conocer más a fondo el contenido de las Observaciones Generales referidas, puede acudir a la página: https://conf-dts1.unog.ch/1\%20SPA/Tradutek/Derechos_hum_Base/ CESCR/00_1_obs_grales_Cte\%20Dchos\%20Ec\%20Soc\%20Cult.html.

9 La página de la Relatora Especial de los Derechos Culturales puede ser consultada en: https:/ / www.ohchr.org/SP/Issues/CulturalRights/Pages/SRCulturalRightsIndex.aspx.

10 Observación General n. ${ }^{\circ} 21$ del Comité de los Derechos Económicos, Sociales y Culturales, respecto del derecho de toda persona a participar en la vida cultural.
}

Villaseñor Anaya, Carlos J. (2021). «El desarrollo sostenible y los derechos culturales». Monograma. Revista Iberoamericana de Cultura y Pensamiento, n. ${ }^{\circ}$ 9, pp. 393-405. doi: 10.36008/monograma.2021.09.2249. http: revistamonograma.com. ISSN: 2603-5839. 
en la gran mayoría de los países; también lo es que en muy pocas ocasiones se ha comprendido a fondo la aportación que tiene el acceso, la participación y la aportación de contenidos simbólicos al logro del desarrollo sostenible; $y$, mucho menos, se ha hecho consciente que los derechos culturales sean indivisibles e interdependientes de derechos fundamentales tan trascendentes como el derechos a la vida, a la libertad o al acceso a la justicia.

Para algunos pareciera un exceso darle esa jerarquía a los derechos culturales, sin darse cuenta de que un ser humano al que se le impide acceder, participar y contribuir a los contenidos simbólicos no contará con los elementos indispensables para representarse a sí mismo, dialogar con los demás y ponerse de acuerdo para construir un proyecto común. Es decir, una ser humano al que se le impiden apropiar contenidos simbólicos es incapaz de desenvolver su naturaleza humana.

En ese orden de ideas, el reconocimiento de los derechos culturales no es una concesión graciosa del legislativo a la sociedad sino, muy contrario, las limitaciones que el Estado impone al gobierno para que los seres humanos cuenten con las garantías necesarias para desplegar su potencial humano.

Inspirados en las ideas de Amartya Sen, podemos decir que gran parte de la libertad de las personas para alcanzar aquello que tienen razones para valorar, está determinada por las posibilidades reales que tengan ellas para el ejercer plenamente sus derechos a acceder, participar y contribuir al desarrollo cultural; a libertad de expresión artística; a gozar de los beneficios morales y materiales de las obras de que sea autor.

Por otra parte, como hemos tratado de demostrar en el cuerpo del presente texto, en tanto que la cultura es ahora reconocida como aquel «conjunto de valores único e irreemplazable, ya que las tradiciones y formas de expresión de cada pueblo constituyen su manera más lograda de estar presente en el mundo»;

Villaseñor Anaya, Carlos J. (2021). «El desarrollo sostenible y los derechos culturales». Monograma. Revista Iberoamericana de Cultura y Pensamiento, n. ${ }^{\circ}$ 9, pp. 393-405. doi: 10.36008/monograma.2021.09.2249. http: revistamonograma.com. ISSN: 2603-5839. 
y, por lo tanto, es la manera en como las personas se piensan a sí mismas, se relacionan con los otros y se ponen de acuerdo para trabajar en conjunto.

En consecuencia, la vertiente cultural es transversal con todos los objetivos y metas del desarrollo sostenible, de donde debe ser respetada la igual dignidad de las culturas y atendida en sus especificidades, a lo largo todas y cada una de las acciones que se desarrollen para su cumplimiento.

Especialmente desde la perspectiva económica, es muy importante comprender que el crecimiento y el combate a la pobreza son acciones que deben encontrar un equilibrio con los aspectos sociales y ambientales del desarrollo; y que, el ejercicio de la libertad cultural es — también- indivisible del desarrollo sostenible.

Vivimos un delicado momento en donde las industrias culturales relacionadas con las nuevas tecnologías se han desarrollado exponencialmente y ahora somos capaces de encontrar abundante ejemplos de cómo es que la necesidad de minimizar los costos de producción, mediante la masificación de los contenidos, con objeto de lograr el máximo rendimiento de las ganancias, está produciendo el fenómeno - ya anunciado por la Declaración de México de 1982 — de la reducción de la diversidad de los contenidos simbólicos y la exclusión de los contenidos de los países en vías de desarrollo.

Lo anterior debe llamar nuestra atención porque está en juego la diversidad creativa de la especie humana y la sostenibilidad del desarrollo; lo cual, en el largo plazo (en el mejor de los casos) llevará a un colapso de las vertientes social y ambiental del desarrollo, sin las cuales la economía se verá radicalmente imposibilitada para prosperar.

Es esta una reflexión que deberá surgir desde los propios agentes de la economía creativa y — sobre la cual— los Estados 
están obligados a emitir las regulaciones normativas que promuevan — tal y como la gran mayoría de ellos se han comprometido a través de la Convención Unesco 2005- que tengan por objeto la promoción y protección de la diversidad de las expresiones culturales.

Esta reflexión es igualmente válida en el proceso de cumplimiento de la Agenda 2030 ONU para el Desarrollo Sostenible.

Hoy mas que nunca es necesario garantizar y promover las condiciones para revitalizar la creatividad social que nos es necesaria para construir nuevos futuros posibles; los cuales, no pueden ser ajenos y mucho menos contrarios, a pleno ejercicio de la libertad para buscar alcanzar aquello que tenemos razones para valorar.

En ese orden de ideas, la economía creativa deberá cumplir su papel facilitador, teniendo siempre en cuenta de que el crecimiento económico no es un fin en sí mismo, sino un medio para desplegar la creatividad de la especie humana, en aras del ejercicio de la libertad y el logro del desarrollo sostenible.

Afortunadamente en auxilio de esa tarea, los países comienzan a desarrollar mecanismos para promover, respetar, proteger y garantizar los derechos humanos culturales, de conformidad con los principios de universalidad, interdependencia, indivisibilidad y progresividad. 